\title{
Considerations for Research and Development of Culturally Relevant Mindfulness Interventions in American Minority Communities
}

\author{
Jeffrey Proulx ${ }^{1}$ (D) Raina Croff ${ }^{1} \cdot$ Barry Oken $^{1} \cdot$ Carolyn M. Aldwin $^{2}$. \\ Crystal Fleming $^{3} \cdot$ Dessa Bergen-Cico ${ }^{4} \cdot$ Thao Le $^{5} \cdot$ Misbah Noorani $^{6}$ \\ Published online: 16 August 2017 \\ (C) Springer Science+Business Media, LLC 2017, Corrected publication September/2017
}

\begin{abstract}
As many health disparities in American minority communities (AMCs) are stress related, there has been an increased interest in the development of mindfulness programs as potential stress-reduction measures in these communities. However, the bulk of the extant literature on mindfulness research and mindfulness interventions is based upon experiences with the larger White community. The intent of this commentary is to share a framework that includes key cultural considerations for conducting research and developing culturally salient mindfulness programs with AMCs. We build on our experiences and the experiences of other researchers who have explored mindfulness in African- and Native American communities; in particular, we examine issues around community outreach with an emphatic gesture toward emphasizing protection of AMCs and their participants. Discussed are considerations with respect to attitudinal foundations in mindfulness-based research and program development with these communities. However, the overall message of this paper is not to provide a "to-do" list of research steps, but to rather, encourage researchers to turn inward and consider the development of skillful characteristics
\end{abstract}

Jeffrey Proulx

proulxj@ohsu.edu

1 Oregon Health \& Science University, 3181 SW Sam Jackson Park Rd, Mail Code: 120, Portland, OR 97239, USA

2 Oregon State University, Corvallis, OR, USA

3 Stony Brook University, Stony Brook, NY, USA

4 Syracuse University, Syracuse, NY, USA

5 University of Hawaii, Manoa, HI, USA

6 Brown University, Providence, RI, USA that will increase the likelihood of a successful research venture while also protecting the cultural traditions of the AMC of interest.

Psychological stress has been shown to be related to poor health outcomes in American minority communities (AMCs) (Goins et al. 2011; Gone 2013; Pouwer et al. 2010). However, daily stress in AMCs may interact with enduring stress stemming from historical trauma(s). Therefore, stress reduction in AMCs should be consistent with long-standing cultural values and reflective of sociocultural historical trajectories of that AMC (Proulx 2010; Sue and Sue 2003).

Research suggests that mindfulness training may be an appropriate and beneficial stress-reduction option for AMCs through the cultivation of insight, peace, and compassion (Pouwer et al. 2010; Woods-Giscombe and Black 2010). We define mindfulness as a quality of conscious awareness, of self and others, that enables one to recognize and understand the nature of thoughts, emotions, and behaviors, thereby freeing oneself from patterns of cognition and behavior that impede optimal mental and physical health (Greeson et al. 2014; Stasiewicz et al. 2012). However, introduction of mindfulness programs to AMCs may be troublesome as White culture, experiences, and social references are embedded in American mindfulness programs. We do not posit that the dominant culture is the backdrop against which all other communities should be viewed, or that there are significant differences in response to mindfulness program across cultural groups. Rather, we are saying that Whites have been the focus of mindfulness research in America, and mindfulness interventions developed for this population should not be applied to AMCs without due sensitivities and cultural considerations.

Awareness of discrimination, bias, and stereotype in oneself, and the effect of racism on others, are considered 
fundamental to psychological and health-related care (Neville et al. 2001; Sue and Sue 2003). Lack of awareness of one's own biases may perpetuate oppression against racial and ethnic minorities (Burkart and Knos 2004). On the other hand, Proulx (2010) noted that the more AMCs are presented with culturally appropriate approaches to healing, the more likely those healthy outcomes associated with the intervention will be enduring (Swinomish Tribal Mental Health Project 1991; Witko 2004). Similarly, the extent to which mindfulness practices can be integrated with traditional treatments will predict the extent to which communities will accept the intervention (Conte et al. 2016; Simonds et al. 2013; Witko 2004). However, the success of this approach should begin with self-reflection of the researcher or clinician mindfulness instructor (see, Proulx 2010; Sue and Sue 2003).

Institutionalized racism is part of the larger structure of racism in which biases are formed, including possible inherent biases in mindfulness instructors. We are not arguing that the current approaches to expanding mindfulness are explicitly discriminatory by any means, but biases often go unrecognized even as they are put into action - as in teaching a mindfulness class. Those actions (which may include how participants are addressed, attitude, language, metaphors, stories, or other means instructors may employ) can create an instruction style and a space that does not allow AMCs to fully enter into the mindfulness class. AMCs have endured cultural loss and intergenerational trauma due to policies and experiences of White cultural expansion in their communities (e.g., suppression of native languages and cultural practices). As such, the steps taken by the mindfulness community may be seen as another example of encouraging AMCs to be more like White communities rather than exploring how spiritual and contemplative traditions in these communities resonate with mindfulness.

On a micro-level, AMCs must remain in a constant state of reinterpretation in order to fit the mindfulness instructions they are receiving into their own cultural framework. While an instructor's actions may not be "against AMCs" per se, they may not be explicitly supportive of their particular cultural frameworks, either. In comparison, persons of the same cultural and historical background as their instructors may be more likely to share common cultural sensibilities, worldview, and value system so that reinterpretation is less necessary. Any level of reinterpretation is extra work for AMCs, and extra work on some level, is stress, even in a mindfulness class trying to develop stress-reducing strategies.

This commentary builds on our experiences creating mindfulness-based research and programming with AMCs. We are guided by lessons from community-based participatory research (CBPR) that asserts engaging with and learning from the community prior to conducting research enhances the likelihood of successful interventions (Simonds et al. 2013). In particular, we focus on our encounters with the Native American and African-American communities. The focus of our research was not simply to assess the effectiveness of mindfulness programs in these specific communities but we were also interested in our ability to use our experiences to develop reflexive and adaptive mindfulness approaches we could use in other AMCs.

We do not expect that what we learned in our research will be generalizable to other communities, but how we learned from these communities could be applied to conducting research with other AMCs. Thus, the experiential lessons we provide here are not meant to be a rigid "todo" list when working in AMCs, but, rather, are intended as thoughtful considerations. We seek to highlight some key cultural considerations researchers need to inform a culturally responsive research program; at the same time, there is ample room for the inclusion of cultural preferences that researchers may discover as they collaborate with their communities of interest. Although our work is influenced by CBPR methodology, this paper is not a review of cross-cultural research or of CBPR methodology, as there are well-detailed sources elsewhere (e.g., Belone et al. 2016). Further, we do not spend time on research design as the focus of this article is on the respectful conduct in research and programmatic development of mindfulness for AMCs.

In the following sections, we list considerations that draw on the experiences of researchers and recommendations provided by community leaders, Institutional Review Board's (IRBs), suggestions from medicine men and women, healers, spiritual leaders, grass-roots and community organizers, and others who have conducted research and delivered clinical mindfulness interventions in these AMCs. We included these considerations as a means to underscore the importance of reaching out to a wide range of spiritual, contemplative, and community leaders in order to integrate mindfulness practices with the cultural strengths of the populations of interest. However, it is important to recognize the influence of Buddhism on mindfulness (Grabovac et al. 2011; Kudesia and Nyima 2015) to be able to better integrate the source of these practices with the AMC populations one wishes to engage with.

We focus on key cultural considerations and frameworks, beginning with a literature review of mindfulness research with Native American and African-American communities. We then outline key processes culled from our experiences developing mindfulness interventions within Native and African-American communities. We conclude with considerations of mindfulness foundations in research that are similarly reflective of mindfulness practices. 


\section{Considerations for Mindfulness in African-American Communities}

African Americans are generally underrepresented in health research, and this extends to mindfulness research. One clear shortcoming is the lack of available culturally specific treatments for stress in African-American communities (Fuchs et al. 2013). The centuries-long history of African Americans being harmed by physicians (Washington 2006) has resulted in high levels of mistrust toward medical professionals (Corbie-Smith et al. 1999). As a result, many African Americans have been reluctant to engage with medical and psychological care, thus underscoring the importance of cultivating trust before introducing any mindfulness-based practices. One way to build trust is to express the importance of the mindfulness intervention as a means of identifying and enhancing cultural strengths.

Stressors and coping processes may differ across cultures (Aldwin 2007; Sue and Sue 2003). Not allowing for historical contexts of stress or experiences that may be unique to AMCs may miss important perspectives from the communities of interest (Fleming 2016). However, emerging research suggests that mindfulness practices, such as mindfulnessbased stress reduction (Kabat-Zinn 2009), breathing awareness meditation (Barnes et al. 2006), and loving kindness meditation (Woods-Giscombe and Black 2010), can improve the physical and/or mental health of AfricanAmerican populations. Yet, this requires openness to cultural perspectives and attentive of fit for different populations. For instance, because many African Americans come from a Christian background, mindfulness practices in Black communities might need to be adapted in ways that are viewed as compatible with biblical principles (Woods-Giscombé and Gaylord 2014). Practices such as benevolence and loving kindness promoted through the practice of metta are harmonious with Christian principles that loving one's neighbor is the most important of directives issued in the Christian tradition. As an illustration, preliminary qualitative data from Proulx et al.'s (2017) study of mindfulness in elder African Americans showed that participants spent class time considering how prayer is a means of "speaking to God" and stillness and meditation are means for better "hearing God."

As noted before, the historical and present contexts of oppression and discrimination that AMCs face are important considerations for mindfulness interventions. Microagressions are commonplace, and intentional and unintentional daily derogatory comments and behaviors experienced by AMCs can lead to stress reactivity that may not be as apparent to other Americans (Harrell et al. 2011; Harwood et al. 2015). AMCs' sensitivity to microaggressions in healthcare contexts may lead to poor coping processes that exacerbate rather than reduce the impact of the stress response (Graham et al. 2013; Masuda et al. 2009; Woods-Giscombe and Black 2010). Prior to engaging in a mindfulness intervention, a deeper conversation with AMCs is needed to explore their perspectives on stress and the development of contextually appropriate coping processes to manage stressors.

\section{Considerations for Mindfulness in Native American Communities}

Mindfulness training may deepen the process of attaining peace and ameliorating poor health and social conditions in Native communities, such as stress, addiction, suicide, and diabetes (Le and Gober 2013; Pember 2012; Zgierska et al. 2009). Further, collaboration with Native communities to develop culturally centered approaches to mindfulness helps to infuse the intervention with meaning, purpose, and identity for Native participants (Swinomish Tribal Mental Health Project 1991). Proulx (2010) noted that the more Native participants can rely on traditional approaches to healing that align with Native culture, the more likely they will negotiate longer-term treatment modalities and express a high sense of self-efficacy necessary for success (Swinomish Tribal Mental Health Project 1991; Witko 2004). Getting participants to enter into mindfulness studies and interventions (Witko 2004) may include the extent to which researchers integrate traditional treatments (e.g., medicine wheel, talking circles) and involve traditional healers (e.g., medicine men and women) in the mindfulness practices.

Croff et al. (2014) found that substance abuse treatment that integrated traditional Native healing practices had increased Native participant engagement. In Le and Gobert's (2013) study on mindfulness and youth suicide prevention with the Salish, Kootenai, and Pend d'Oreille tribes in Montana, they specifically elicited elders' blessings and incorporated input and stories from native story tellers, such as the careful attention of picking berries and the infusion of one's focus and spirit into making moccasin. This allowed for ease of participant recruitment and collaboration with the tribal school to conduct the intervention. Additionally, the respect for and integration of traditional ways facilitated sustainability and continuation after study completion. In fact, mindfulness continues to be infused and incorporated into many facets of tribal social service programs in the groups studied by Le and Gobert (2013), with tribal leaders and their support staff attending conferences, trainings, and workshops to build tribal human and social capital in mindfulness. Hence, it is important that researchers outside the Native community reach out to traditional healers to fully understand how traditional treatments are implemented. Native people will quickly sense a misguided attempt to use their traditional healing methods, which can lead to an unsuccessful research project 
and a negative legacy that could impair future research in that community.

Traditional elements of resilience should not be peripheral to mindfulness research and clinical interventions (Proulx 2010). Instead, traditional elements should constitute the core values of mindfulness practices, and researchers should consider where mindfulness-based practices (e.g., Kabat-Zinn 2009) overlap with Native traditional healing practices (Le and Proulx 2015). Further, commonalities between mindfulness and Native traditional practices should take a culturefocused approach and community ownership, such as that illustrated in Le and Gobert (2013). However, mindfulness researchers working in Native communities need to also be aware of the diversity of attitudes across Native cultures and accept the fact that there is not one stereotypical Native American (see Proulx 2010).

Different generations of Native people will be at different stages of acculturation (e.g., assimilation, integration) to dominant American society and may represent a spectrum of acceptance of dominant culture perspectives (Witko 2004). Further, developing a culturally specific mindfulness program for Natives living in urban settings poses a challenge because of the heterogeneity of nations represented in the metropolitan milieu. Working with different generations in urban settings may further complicate the implementation of culturally based models of mindfulness. For example, the choice of whether or not to use substance abuse treatments that integrate traditional Native practices was a source of tension between acculturated urban Native youth and their reservation-born-and-raised parents who preferred traditional care models (Croff et al. 2014).

As with African-American communities, many Native American populations rely heavily on Christian churches for spiritual guidance. In our experience, this has presented some barriers to recruitment in certain communities and needs to be considered at length prior to engaging in the mindfulness intervention. This highlights the importance of developing strong networks of community advisors within the Native community prior to proposing an intervention or study. Having these key players inform and guide the study from the start provided two key components: (1) building AMC trust through association with these community members and (2) guidance for recruiting and consent form language. This information is critical to building trust and dialog in a community that may have received messages that mindfulness (and/or academic/medical research) is somehow counter to AMC cultural values. However, mapping concepts found in Buddhism and mindfulness practices onto concepts promoted in Christianity, such as developing respect for all life by reducing aggression and hatred in the mind (Mikulas 2015), may help to bridge varying spiritual and contemplative backgrounds.

The following considerations for research and clinical interventions that highlight mindfulness are partially based on the extant literature. However, we illustrate these concepts through our own experiences and show how we were able to overcome barriers to conducting research and developing trust in our communities of interest. Notably, suggestions from previous researchers, community organizers, and others, including community members we worked with, as well as our own experiences as people from AMCs, have informed this work. Several of these actors suggested that we do not apply their solutions in exactly the same way they did but instead use them as guides and adapt them to the situations we encountered.

\section{Foundations for Mindfulness Research in American Minority Communities}

Kabat-Zinn (2009) encourages the incorporation and use of the attitudinal foundations such as trust, gratitude, and beginner's mind, when engaging in mindfulness practice. We have found that these attitudinal foundations are equally important for ethical and effective research, particularly with AMCs. In the following section, we illustrate the application of some of these attitudinal foundations when conducting research with AMCs. These points are particularly salient as our experiences show that the qualities of the researcher himself or herself are as important as the quality of the research. Therefore, we hope that these considerations will help researchers to internalize specific characteristics that will increase the likelihood of acceptance of the research as well as protect the communities of interest.

\section{Trust}

In many instances, communities explicitly told us that trust would be critical to the success of our projects. Our populations gave several explicit and implicit signals that we should work hard to develop and maintain that trust, but it was clear early on that developing trust was not an easy task. Much of this is due to distrust of the health care system (including mental health), which can prove to be an obstacle to mindfulness research (Fuchs et al. 2013). This is especially true when considering that mindfulness interventions are often framed in relation to existing mental health paradigms, which makes the harmful assumption that AMCs are as familiar and comfortable with these references as their White counterparts. It has been shown that researchers and health providers are most effective when they are able to shift their therapeutic focus to meet the needs of their participants and/or clients, rather than neglecting to do so in the service of rigidly adhering to research and treatment based on dominant society perspectives (Sue and Sue 2003). As an extrapolation, we posit that it is the responsibility of the researcher to consider the 
interests of the participants and ensure that they are prioritized over the research.

We urge scholars conducting research on mindfulness, as well as mindfulness practitioners, to be aware of the diversity of perspectives that exist within African-American, Native American, and other AMCs. Accounting for this diversity requires adopting an intersectional approach modeled after the contributions of Black feminist theory (Crenshaw 1989). Intersectionality (Crenshaw 1991) suggests that forms of social domination (racial, gender, class, etc.) are not isolated from one another. Rather, they are interlocked and inextricably linked (Cooper 2016). Concerning mindfulness research, scholars should consider the possibility that AMCs may have different experiences with mindbody interventions depending on their gender, sexuality, class, and personal histories. For example, participants in Proulx et al.'s (2017) study of mindfulness in AfricanAmerican communities noted that that being in the center of family and social life further exacerbated the underlying stresses associated with racial history in the United States and of aging. Understanding how these roles intersect can help in the development of and conversation about mindfulness in participants' lives.

Le and Gobert's (2013) mindfulness study in Native communities addressed the aforementioned trust concerns by having the principal investigator develop a relationship with a community champion. This community champion was an early adopter of mindfulness concepts and programs and she became a trusted advocate with elders and the tribal council. The principal investigator was in direct communication with the champion in the day-to-day affairs of the study. In turn, the champion provided periodic study updates to elders and the tribal council. Through this continuous exchange of information, trust developed and continued throughout the study and continued with the champion's successor who replaced her at tribal social services. Further, this interaction with the tribe provided the research team with a better understanding of how variables often associated with inequity (as mentioned above) map onto the concept of intersectionality where being Native and a woman or being Native and poor may change the of mindfulness approach to be introduced. Going to these lengths showed the commitment of the research team to protect and better understand the complex dynamics that are more prevalent in AMCs than in the dominant society.

\section{Research Involving Biological Sampling}

Research in AMCs has an unsavory history, including involuntary experimentation (Dula 1994) and unethical withholding of treatment (as in the case of the Tuskegee Experiment), as well as misuse of biological sampling materials (Bussey-Jones et al. 2010). We wish to highlight the importance of being sensitive to the protection of sacred knowledge and beliefs, including culturally differing views of the body and bodily fluids. Interviews with our community partners revealed suspicion, bordering on resentment, of the academy's efforts to include AMCs in research, specifically when the research includes biological sampling. We use the example of biological sampling to illustrate how accepted practices in medical and academic research (e.g., consent language for biomarker collection that reflects the total uncertainty of when and by whom their samples will be used) may not be acceptable to AMCs because of unethical health research in the past. Clarifying what consent allows for, including what biological samples may be stored in a repository and potentially re-used in the future, may help to reduce confusion. Clearly spelling out sampling procedures before presenting to the tribal board and African-American advisors was helpful in securing permission to advance our research approvals.

As we prepared an IRB proposal for presentation to a tribal board, our attention was drawn to a lawsuit between a Native community and a major university. This lawsuit addressed researchers' reuse of biological samples without explicit tribal permission (Mello and Wolf 2010). This was concerning to us because we proposed to use biological sampling to measure changes in stress hormone activity before and after the mindfulness intervention. To address potential legal concerns, we reached out to local researchers and biomarker research companies to assess how we could address tribal concerns about biomarker analysis. They advised us to emphasize that our research used saliva, not blood or tissue sampling, and demonstrate our commitment to protecting participants with detailed information on participant confidentiality and anonymity safeguards for salivary samples and results. Further, we emphasized that our biomarker analysis agreement included a clause to destroy all samples immediately after completion of biomarker assays. This experience is but one example of the potential benefit that forethought and cultural sensitivity bring to community and to research trajectory.

\section{Importance of Language and the Process of Consent}

Experienced researchers know there are at least two levels of consent that must be approved for human subject research: IRB review and approval and participant consent. Research with AMCs may also require additional levels of review and consent, such as a tribal IRB, and/or informal or formal community committees or gatekeepers. The language in consent forms and research instructions that are not attentive to cultural norms may dissuade gatekeepers from allowing research to move forward. However, an attitude of humility and compliance can go a long way in turning a rejected proposal into an opportunity for gatekeepers to teach researchers about how to design their study better. 
It should be made clear to AMCs that researchers are an advocate for the primacy and privacy of the community. At several junctures, we had to choose whether to move forward with research because of seemingly intractable differences in what institutional (university) IRB required versus what our African-American community advisors and tribal IRBs were willing to allow. This last illustration may be particularly salient for studies that require HIPAA forms to be signed. In general, we adapted our proposals to meet university and community requirements while maintaining a protective stance for our communities of interest. This is where our knowledge of AMCs proved effective in that we were able to convey our intention to protect the needs and concerns of these communities to academic administrations. Notably, this information and our advocacy for our communities were sufficient for our review boards to work with us to adapt our consent forms for the protection of our participants.

We made concrete efforts to show that we did not intend to collect data and then disappear, a common concern for AMCs. Instead, we emphasized that, after collecting data, we would return to the communities we were studying in order to get their help interpret the results and decide where to submit for publication. In this way, we were able to assuage fears that our results were not truly representative of cultural beliefs. This was paramount: making clear that our desire was to show how AMC experiences could inform the mindfulness community, as much as how mindfulness could affect overall wellbeing in $A M C s$. Further, we made a visible effort to have a principal investigator present at tribal IRB meetings and at AfricanAmerican community meetings to show our intention to be accessible and community-driven beyond our research project. At meetings, we witnessed several occasions where researchers and their institutions were scolded because principal investigators did not attend IRB or organizational meetings, or sent proxies instead. In another instance, we built trust and buy-in when a member of our research team was invited to speak to a Sunday congregation at a Black church (at the invitation of the pastor) regarding our mindfulness study. Needless to say, we received numerous participant requests at the end of the service, and it did help that the sermon topic that week was about stress.

\section{Gratitude}

We found from previous researchers that a demonstration of thankfulness to the community was necessary to move research forward. In one form or another, we were often asked, "How will your research benefit our community in the longrun?" Researchers must come to communities prepared to explain how the mindfulness-based program may benefit the community's mental and spiritual health, and plans for sustainability beyond the research project. An earnest effort to develop a foundation in the mindfulness research that has the potential to have an enduring benefit to the AMC will perceived as an act of gratitude and generosity.

\section{Humbly Emphasize What Your Research Will Contribute to the Community}

As noted above, AMCs are concerned that researchers will enter the community only to leave without any enduring benefit from the research. Early on, community members told us that this concern is not insignificant and that we had to make explicit efforts to learn about the community needs and how we might procure the materials to meet those needs. In our Native research, we were led to a previous researcher whose approach to giving back to the community was to develop and maintain an alternative school that employed tribal and progressive educational methodologies to teach Native youth. This knowledge helped us understand the extent to which researchers try to fulfill community needs and sustain the researcher-community relationship.

We made connections with the Native school and discussed plans to apply lessons learned in mindfulness research in elementary and high schools (Black and Fernando 2014) to develop programs designed to address high rates of strain in Native youth (Le and Gobert 2013). The tribal IRB supported this particular proposal and it has led to a relationship where we are currently discussing options for mindfulness studies in other aspects of that community (such as mindfulness for Native schoolchildren). It was this sensitivity and willingness to be generous that paved the way for future potential mindfulness studies with this particular community.

Similarly, we asked our elder African-American advisors to suggest ways we might meaningfully contribute to the community beyond the scope of the immediate research project. In these discussions, we ascertained that the community group we wanted to partner with did not have a strong web presence with which to communicate with their members. When we met with the community action group, as we anticipated, they asked, "How will your research benefit our community in the long-run?" We were ready with some preliminary ideas, including an offer to help the community group develop a more robust online presence in order to better communicate with their members. We were also transparent that, in improving their website and listserv, this would also benefit the project's recruitment outreach. Our research project had the intern personnel, expertise, and funding to fulfill this need, thereby establishing a transactional relationship between the research team and the community.

Using the action group's preferred network, we reached out to students from AMCs to fill internship positions, which the action group and the research team saw as a means of greater inclusiveness within the community. Both parties recognized that every research project brings with it the opportunity to further the academic career or provide experience for a student 
from an AMC background. Further, we cannot expect that AMC students to know how to find us, whereas higher socioeconomic White students may already have increased access than students from AMCs to the academic channels of opportunity - whether through family, friends, or colleagues. Researchers working with AMCs must make deliberate efforts to seek out students from AMCs for internship positions and cannot simply accept the first qualified students who find them. Every community we worked with applauded our efforts to identify and recruit these students/interns.

\section{Beginner's Mind Approach to Research in African- and Native American Communities}

Research with AMCs requires academic researchers to suspend their well-earned expertise to a certain extent. Although our experiences may suggest that mindfulness interventions in AMCs not yet practicing will be beneficial, it is important to understand that the causes and outcomes of stress and suffering differ among communities. Further, it is important to understand that communities have well-developed coping mechanisms that are culturally specific and recognizable by community members. Reliance on our own culturally biased experiences may lead researchers to miss important opportunities to learn from the community and to develop appropriate mindfulness interventions. This is true regardless if researcher/instructor ethnicity matches the community of study; there may still be miscommunication, even though researcher/instructors may share racial and ethnic backgrounds with the participant/student. Thus, we put forth illustrations of the importance of approaching research with a beginner's mind, such as allowing the community to teach the researcher about community strengths and culture, as well as meeting the participants where they are. This requires being a reflective practitioner, revising curriculum language, responding to the participants as necessary, and taking the other person's perspective.

\section{Drawing on Existing Community Strengths}

Cultural and community strengths are consistent themes in studies of African- and Native American resilience (Abdou 2014; Gone 2009). Relying on community advisors/elders to provide direction for stress-reduction interventions may provide the best option for adapting mindfulness classes. This means that stress-reduction interventions must seek community input to meet needs of each specific community. Efforts must be made to familiarize oneself with the culture of the community of interest in order to better understand and internalize the history and worldview of these populations. Le and Proulx (2015) illustrated this in a study that incorporated the Native Hawaiian/Pacific Islander cultural concept of
Aloha (as presence in the breath) in the mindfulness class with incarcerated youth. They found Native Hawaiian/Pacific Islander youth to be accepting of mindfulness practices as it resonated with their cultural understanding, and that the class resulted in lower stress perception, more skillful responses to stress, and favorable cortisol and immune system responses to stress pre- and post-training.

As previously noted, several people in the communities we worked with have expressed being unimpressed by interventions offered to their communities because they were not responsive to their community's cultural perspectives. Taking heed, we often used qualitative focus groups and individual interviews to gather cultural knowledge as communicated by community members in their own words rather than relying on our own assumptions or interpretive language. Prior to designing our qualitative questions, we asked our oversight committees to provide us with a list of ten stressors they experienced personally or knew other community members experienced. The specific answers are mostly outside the scope of this paper, and each community will have distinct stressors. Nonetheless, it is important to note that some of the answers were unexpected and were otherwise unaddressed by many stressor measures common in research.

One of our African-American mindfulness groups discussed how displacement from historically Black neighborhoods, including more recent rapid gentrification, has had a major effect on the African-American community in that area. This point is important: researchers who have not personally experienced this historical background are less likely to understand how historical context interacts with daily stressors to lead to perceived or real stressful encounters unique to these communities. More importantly, a teacher/researcher's lack of personal experience with these contexts may become painfully obvious to everyone involved and reduce the effectiveness of the intervention.

This point cannot be overstated: a person of color, preferably from the community one is studying, should take a leadership role in the development of the mindfulness-based interventions and the translation of research methods to be conducted with the community. Accomplishing this goal may not be easy; minorities make up a small percentage of mindfulness-based practitioners and participants (WoodsGiscombé and Gaylord 2014). However, we expect that this will change with wider acceptance of mindfulness practices in these communities; and we expect that wider acceptance will come as researchers apply more culturally minded approaches in line with the specific communities they wish to serve.

\section{Learning About the Culture and Meeting Participants Where They Are}

It is important that researchers demonstrate knowledge of the traditions and history of the communities with which they 
seek to collaborate. Blum (2014) noted that people from AMCs might be less likely to remain in meditation programs if they do not feel there is cultural congruence between the mindfulness intervention and cultural concepts familiar to them. The Mindful Allies program mentioned by Blum included strategies that emphasized meeting people where they are, specifically, by using existing outreach methods familiar to that community.

Our community advisors suggested that we include funding for gas for transportation to and from the mindfulness class. Although we spent time pulling together funding for participant compensation (e.g., for orientation and preliminary data collection, participation in focus groups, home saliva collection, completion bonus), we did not take into account something as fundamental to the project's success as the cost of getting to and from the mindfulness classroom. Blum (2014) highlighted these considerations and mentioned that the Mindful Allies program provided childcare and dinner on-site during the mindfulness sessions. Our collaborating community explicitly told us that not taking such approaches to maximize participant convenience would indicate that we were not adequately prepared to meet the community where they are. The Insight Meditation Society (2016) pointed out those efforts to ensure convenience sends the message that these interventions are not just another predominately White institution proffering healing to AMCs but indicates an effort to blend with the community.

We reached out to Native and African-American researchers and healers to learn about possible connections between Native traditions and mindfulness interventions. Notably, our collaborators in the African-American community emphasized turning away from the terms "meditation" and "mindfulness" during the recruiting process and instead use the term "stress reduction." Further, we strived to communicate that our intervention reflected the community's culture, beliefs, and worldview. Nonetheless, the conception that mindfulness is a White practice using Eastern traditions was deeply rooted. This way of seeing mindfulness may be problematic when tailoring an intervention to resonate with community members of various ages, as there may be a generational divide on the acceptance of non-Christian traditions as they pertain to spiritual healing. However, Proulx and Aldwin (2016) noted that coping with stress may have different connotations across cultures, but that finding the existing cultural strengths (e.g., the Christian Church, family, and community networks) that map onto mindfulness practices, may prove potent in encouraging people from AMCs to enter into mindfulness interventions. However, we did not obscure the fact that the mindfulness practices presented were based in Eastern traditions and included a general background of how the practices we presented were developed over time. This provided for full disclosure and opened the conversation to any discomfort people may have had with the perception or history of mindfulness practices (and an opportunity to discuss discomfort).

A traditional Native healer encouraged us to take a Native view of guidance and success in our project. For example, she encouraged us to continually ground ourselves (which is similar to mindfulness practices) by paying attention to the bottom of our feet as they meet the Earth. Further, she told us to look to nature for signs of our progress. These considerations are much different from the signposts researchers are usually familiar with, such as grant funding, IRB approval, publication, etc. We were told that these aspects of our work are important but to turn to the Native ways of learning and healing ourselves prior to reaching out to the Navajo people.

\section{Conclusion}

The core issue is not merely that "White values" are infused in mindfulness in the West, rather, mindfulness practices are taking place within contexts of power and inequality that are part of the American story. Therefore, when White culture informs mindfulness, that influence needs to be read in relation to racial and interrelated forms of privilege more broadly. We know that when one demographic is overrepresented in anything, the work in that field will typically be biased in ways that reflect the values and practices of that demographic.

Researchers must recognize cultural gaps between their own assumptions and the reality of cultural perspectives as communicated by AMCs. This includes a wholehearted effort to compensate for their lack of cultural understanding, including using researchers whose ethnicity matches the community of interest. Ultimately, this merits a discussion with collaborating community members about how narrowly to define the "community" of interest (i.e., African Americans in Oregon; African Americans in Portland, Oregon; older African Americans in Portland Oregon). A community may share vast cultural perspectives yet also maintain a diversity of major and nuanced cultural differences influenced by region, status, age, and other social-demographic and historical factors. Researchers can establish and maintain a relationship with community members in a number of ways, including withholding personal assumptions, basing decision making in collaboration with community members, careful research into the community's context, and using the collaborative approaches promoted in CBPR literature (Simonds et al. 2013),

In this article, we illustrated several considerations for conducting mindfulness research in AMCs:

1. Include people from AMCs of interest and provide them with primary and visible roles in the research from the conception of the research problem to the interpretation of results; 
2. Learn about the culture under consideration with emphasis on qualitative inquiry with community members;

3. Understand the history of research in the community of interest, including any sustained efforts to maintain community integrity;

4. Ask the community what their needs are and emphasize how the research will contribute to the community needs;

5. Work with a small group of advisors from the community who are generally known within the community as community gatekeepers;

6. Interact often and genuinely with the community groups who are allies and/or advisors;

7. Understand and internalize to the best of your ability, the cultural strengths of the community of interest; and

8. Emphasize beginner's mind and gratitude.

These considerations are not exhaustive; we will likely learn more as research in this field expands.

We found it important to cultivate the characteristics that our communities noted as important to them. These may be key questions to ask oneself: Am I worthy of gaining trust in this community? Am I worthy of receiving valued knowledge from these communities? Can I sustain what I intend to start? Am I willing to change the grant/ protocol/research team to suit the needs of the community as dictated by them? Often the measures one needs to show worthiness are included in the directions listed in the community's IRB proposal or stated in discussion with community advisors. Sometimes one needs to ask for direction. Either way, it became clear to us that the populations we worked with could tell the difference between researchers that expended the effort to achieve worthiness and acceptance, and those who did not.

Reflecting on the attitudinal foundations of mindfulnessbased research, we strongly encourage researchers to be cognizant and embody trust, gratitude, patience, beginner's mind, curiosity, non-judging, acceptance, and non-attachment in their research ethos. These are not necessarily linear foundations but overlapping concentric ways of being that must be continuously considered. Researchers have a responsibility to build trust with the community and sustain it, to consistently re-examine the relevant power structures and their own intentions, and to consider who stands to benefit from the research.

\section{Compliance with Ethical Standards}

Conflicts of Interest All procedures performed in studies involving human participants were in accordance with the ethical standards of the institutional and/or national research committee and with the 1964 Helsinki declaration and its later amendments or comparable ethical standards. This article does not contain any studies with animals performed by any of the authors. Informed consent from individual participants was obtained from subjects in each study included in this paper.

\section{References}

Abdou, C. (2014). Minority aging before birth and beyond: Life span and intergenerational adaptation through positive resources. In $\mathrm{K}$. Whitfield \& T. Baker (Eds.), Handbook of minority aging (pp. 9-24). New York: Springer Publishing Company.

Aldwin, C. M. (2007). Stress, coping and development: An integrative perspective. New York: Guilford.

Belone, L., Lucero, J. E., Duran, B., Tafoya, G., Baker, E. A., Chan, D.,.. Wallerstein, N. (2016). Community-based participatory research conceptual model: Community partner consultation and face validity. Qualitative Health Research, 26(1), 117-135. doi: 10.1177/ 1049732314557084.

Black, D. S., \& Fernando, R. (2014). Mindfulness training and classroom behavior among lower-income and minority elementary school children. Journal of Child and Family Studies, 23(7), 1242-1246. doi: 10.1007/s10826-013-9784-4.

Blum, H. (2014). Mindfulness equity and Western Buddhism: Reaching people of low socioeconomic status and people of color. International Journal of Dharma Studies, 2(10), 1-18. doi:10.1186/s40613-014-0010-0.

Bussey-Jones, J., Garrett, J., Henderson, G., Moloney, M., Blumenthal, C., \& Corbie-Smith, G. (2010). The role of race and trust in tissue/ blood donation for genetic research. Genetics in Medicine, 12(2), 116-121. doi:10.1097/gim.0b013e3181cd6689.

Conte, K. P., Schure, M. B., \& Goins, R. T. (2016). Older American Indians' perspectives on health, arthritis, and physical activity: Implications for adapting evidence based interventions. Prevention of Chronic Disease, 13, E81. 160098. doi:10.5888/pcd13.160098.

Cooper, B. (2016). Intersectonality. In L. Disch \& M. Hawkesworth (Eds.), The Oxford handbook of feminist theory. London: Oxford University Press.

Corbie-Smith, G., Thomas, S., Williams, M., \& Moody-Ayers, S. (1999). Attitudes and beliefs of Americans toward participation in medical research. Journal of General Internal Medicine, 14(9), 537-546. doi:10.1046/j.1525-1497.1999.07048.x.

Crenshaw, K. (1989). Demarginalizing the intersection of race and sex: A black feminist critique of antidiscrimination doctrine, feminist theory, and antiracist politics. University of Chicago Legal Forum, 140(1), 139-167.

Crenshaw, K. (1991). Mapping the margins: Intersectionality, identity politics, and violence against women of color. Stanford Law Review, 43(6), 1241-1299. doi:10.2307/1229039.

Croff, R. L., Rieckmann, T. R., \& Spence, J. D. J. (2014). Provider and state perspectives on implementing cultural-based models of care for American Indians and Alaska Native patients with substance use disorders. Journal of Behavioral Health Services \& Research, 41(1), 64-79. doi:10.1007/s11414-013-9322-6.

Dula, A. (1994). African American suspicion on the healthcare system is justified: What do we do about it? Cambridge Quarterly of Healthcare Ethics, 3(3), 247-257. doi:10.1017/s0963180100005168.

Fleming, C. M. (2016). Spirituality and mindfulness. In J. C. Nash (Ed.), Gender: Love. Farmington Hills, MI: Macmillan Reference USA.

Fuchs, C., Lee, J. K., Roemer, L., \& Orsillo, S. M. (2013). Using mindfulness-and acceptance-based treatments with clients from nondominant cultural and/or marginalized backgrounds: Clinical considerations, meta-analysis findings, and introduction to the special series: Clinical considerations in using acceptance-and mindfulness-based treatments with diverse populations. Cognitive and Behavioral Practice, 20(1), 1-12. doi:10.1016/j.cbpra.2011.12.004.

Goins, R. T., Garroutte, E. M., Fox, S. L., Dee Geiger, S., \& Manson, S. M. (2011). Theory and practice in participatory research; lessons from the Native elder care study. The Gerontologist, 51(3), 285-294. doi: 10.1093/geront/gnq130. 
Gone, J. P. (2009). A community-based treatment for Native American historical trauma: Prospects for evidence-based practice. Journal of Counseling and Clinical Psychology, 77(4), 751-762. doi:10.1037/a0015390.

Gone, J. P. (2013). A community-based treatment for Native American historical trauma: Prospects for evidence-based practice. Spirituality in Clinical Practice, 1(S), 78-94. doi:10.1037/a0015390.

Grabovac, A. D., Lau, M. A., \& Willett, B. R. (2011). Mechanisms of mindfulness: A Buddhist psychological model. Mindfulness, 2(3), 154-166. doi:10.1007/s12671-011-0054-5.

Graham, J. R., West, L. M., \& Romer, L. (2013). The experience of racism and anxiety symptoms in an African American sample: Moderating effects of trait mindfulness. Mindfulness, 4(4), 332 341. doi:10.1007/s12671-012-0133-2.

Greeson, J., Garland, E., \& Black, D. S. (2014). Mindfulness: A transtherapeutic approach for trans-diagnostic mental processes. In A. Ie, C. Ngnoumen, \& E. Langer (Eds.), The Wiley Blackwell handbook of mindfulness volume II (pp. 533-562). Oxford: Wiley Blackwell.

Harrell, C. J. P., Burford, T. I., Cage, B. N., Nelson, T. M., Shearon, S., Thompson, A., \& Green, S. (2011). Multiple pathways linking racism to health outcomes. Du Bois Review: Social Science Research on Race, 8(01), 143-157. doi:10.1017/s1742058x11000178.

Harwood, S. A., Choi, S., Orozco, M., Browne Huntt, M., \& Mendenhall, R. (2015). Racial microaggressions at the University of Illinois at Urbana-Champaign: Voices of students of color in the classroom. University of Illinois at Urbana-Champaign. Accessed 25 Mar 2017 http://works.bepress.com/stacy_harwood/5/.

Insight Meditation Society. (2016). Commitment to diversity. N.p., n.d. Web. 24 June 2016. https://www.dharma.org/about-us/diversity

Kabat-Zinn, J. (2009). Full catastrophe living: using the wisdom of your body and mind to face stress, pain, and illness (15th anniversary edn.). New York: Delta Trade Paperback/Bantam Dell.

Kudesia, R. S., \& Nyima, V. T. (2015). Mindfulness contextualized: An integration of Buddhist and neuropsychological approaches to cognition. Mindfulness, 6(4), 910-925 https://doi.org/10.1007/s12671014-0337-8.

Le, T., \& Gobert, J. (2013). Translating and implementing a mindfulness-based youth suicide prevention intervention in a Native American community. Journal of Child and Family Studies, 24(1), 12-23. doi:10.1007/s10826-013-9809-z.

Le, T., \& Proulx, J. (2015). Feasibility of mindfulness-based intervention for incarcerated mixed-ethnic/Native Hawaiian Pacific Islander youth. Journal of Asian American Psychology, 6(2), 181-189. doi:10.1037/aap0000019.

Masuda, A., Anderson, P. L., \& Sheehan, S. T. (2009). Mindfulness and mental health among African American college students. Complementary Health Practice Review, 14(3), 115-127. doi:10. 1177/1533210110363893.

Mello, M. M., \& Wolf, L. E. (2010). The Havasupai Indian tribal caselessons for research involving storage of biological samples. The New England Journal of Medicine, 363, 204-207. doi:10.1056/ NEJMp1005203.

Mikulas, W. L. (2015). Ethics in Buddhist training. Mindfulness, 6(1), 14-16. doi:10.1007/s12671-014-0371-6.
Neville, H. A., Worthington, R. L., \& Spanierman, L. B. (2001). Race, power, and multicultural counseling psychology: Understanding white privilege and color-blind racial attitudes. In J. G. Ponterotto, J. M. Casas, L. A. Suzuki, \& C. M. Alexander (Eds.), Handbook of multicultural counseling (2nd Ed) (pp. 257-288). Thousand Oaks, CA: Sage.

Pember, M. (2012). Relax, just do it. Indian Country Today, 2(3), 34-35.

Pouwer, F., Kupper, N., \& Adriaanse, M. C. (2010). Does emotional stress cause type 2 diabetes mellitus? A review from the European Depression in Diabetes (EDID) Research Consortium. Discovery Medicine, 9(45), 112-118.

Proulx, J. (2010). Review of Native American access to mental health care in Northern California and Oregon. McNair Scholars Journal, $6,52-58$.

Proulx, J., \& Aldwin, C. M. (2016). Stress and coping theory in geropsychology. In N. A. Pachana (Ed.), Encyclopedia of Geropsychology. New Delhi: Springer. doi:10.1007/978-981-287080-3 120-1.

Proulx, J., Croff, R., Hebert, M., \& Oken, B. (2017). Results of a mindfulness intervention feasibility study among elder African American Women. Unpublished Raw Data.

Simonds, V. W., Wallerstein, N., Duran, B., \& Villegas, M. (2013). Community-based participatory research: Its role in future cancer research and public health practice. Prevention of Chronic Disease, 10, 1-7. doi:10.5888/pcd10.120205.

Stasiewicz, P. R., Bradizza, C. M., Gudleski, G. D., Coffey, S. F., Schlauch, R. C., Bailey, S. T., Bole, C. W., \& Gulliver, S. B. (2012). The relationship of alexithymia to emotional dysregulation within an alcohol dependent treatment sample. Addictive Behaviors, 37(4), 469-476. doi:10.1016/j.addbeh.2011.12.011.

Sue, D. W., \& Sue, D. (2003). Counseling the culturally diverse: Theory and practice (4th ed.). New York: Wiley.

Swinomish Tribal Mental Health Project. (1991). A gathering of wisdoms. Mt. Vernon, WA: Swinomish Tribal Community.

Washington, H. A. (2006). Medical apartheid: The dark history of medical experimentation on black Americans from colonial times to the present. New York, NY: Anchor Books.

Witko, T. M. (Ed.). (2004). Mental health care for urban Indians: Clinical insights from Native practitioners. Washington, DC: American Psychological Association.

Woods-Giscombe, C. L., \& Black, A. R. (2010). Mind-body intervention to reduce risk for health disparities related to stress and strength among African American women: The potential of mindfulness-based stress reduction, loving-kindness, and the NTU therapeutic framework. Complementary Health Practice Review, 15(3), 115-131.

Woods-Giscombé, C. L., \& Gaylord, S. A. (2014). The cultural relevance of mindfulness meditation as a health intervention for African Americans: Implications for reducing stress-related health disparities. Journal of Holistic Nursing, 32, 147-160. doi:10.1177/ 0898010113519010.

Zgierska, A., Rabago, D., Chawla, N., Kushner, K., Koehler, R., \& Marlatt, A. (2009). Mindfulness meditation for substance use disorders: A systematic review. Substance Abuse, 30(4), 266-294. doi:10.1080/08897070903250019. 